\title{
Regulation of p63 expression in primary and immortalized nasopharyngeal epithelial cells
}

\author{
Y.L. YIP and S.W. TSAO \\ Cancer Biology Laboratory, Department of Anatomy, Li Ka Shing Faculty of Medicine, \\ The University of Hong Kong, Pokfulam, Hong Kong SAR, P.R. China
}

Received April 7, 2008; Accepted May 26, 2008

DOI: 10.3892/ijo_00000057

\begin{abstract}
Mutation of the p53 gene is a common event in human cancer. Interestingly, p53 mutation is uncommon in nasopharyngeal carcinoma (NPC). The $\triangle \mathrm{Np} 63$ has been postulated to have a dominant-negative effect on the function of the p53 gene and may play a role in the pathogenesis of nasopharyngeal carcinoma. Immortalization is a common property of cancer cells and is believed to be an early event in carcinogenesis. At present, the relationship between $\Delta \mathrm{Np} 63$ and immortalization is poorly understood. In this study, we defined the expression profile of p63 and its various isoforms in primary and immortalized nasopharyngeal epithelial cells. Also, we elucidated some events regulating the expression of p63. Elevated expression of p63 was generally detected in both primary and immortalized nasopharyngeal epithelial cells at their proliferation stage and the predominant isoform of p63 expressed was $\Delta \mathrm{Np} 63 \alpha$. p63 expression was suppressed upon cellular senescence of primary nasopharyngeal epithelial cells and induction of terminal differentiation in immortalized nasopharyngeal epithelial cells. Expression of $\Delta \mathrm{Np} 63$ alone was able to drive clonal proliferation in primary nasopharyngeal cells in culture while downregulation of $\Delta \mathrm{Np} 63$ induced cellular apoptosis. All these results support a role of $\Delta \mathrm{Np} 63$ in proliferation and immortalization which facilitates pathogenesis of nasopharyngeal carcinoma. TGFß and retinoic acid downregulated the expression of p63 in immortalized nasopharyngeal epithelial cells and may play a role in regulating differentiation in squamous epithelial cells with potential applications in prevention and treatment of nasopharyngeal carcinoma.
\end{abstract}

Correspondence to: Professor George S.W. Tsao, Department of Anatomy, Room 1-54, Li Ka Shing Faculty of Medicine, The University of Hong Kong, 21 Sassoon Road, Pokfulam, Hong Kong SAR, P.R. China

E-mail: gswtsao@hkucc.hku.hk

Key words: p63, differentiation, immortalization, nasopharyngeal epithelial cells, proliferation, senescence

\section{Introduction}

The $p 63$ gene is a member of the $p 53$ family and shares similar structure homology and functions with other $p 53$ family members including $p 73(1,2)$. Two major classes of p63 isoforms have been identified: TAp63, which is the full-length transactivating isoform, and the $\Delta \mathrm{Np} 63$, which is truncated at the $\mathrm{NH}_{2}$-terminal transactivation domain. The TAp63 and $\Delta \mathrm{Np} 63$ isoforms are further divided into 3 subtypes, $\alpha, \beta$ and $\gamma$. The crucial role of $p 53$ in regulation of cell cycle and apoptosis is well-documented. The TAp63 isoforms have similar functions to the wild-type $\mathrm{p} 53$ protein and induce apoptosis in cells, while the $\Delta \mathrm{Np} 63$ isoforms could not transactivate many of the $\mathrm{p} 53$ responsive genes and may function in a dominant-negative way to modulate p53 function in cells (3). The $\Delta \mathrm{Np} 63$ is known to be involved in the development and maintenance of epithelial structures and is highly expressed in the proliferative keratinocytes at the basal layer of skin epidermis. A recent study showed that $\Delta$ Np63 induces expression of $\alpha 6 \beta 4$ in skin keratinocytes and plays a crucial role in the attachment of the basal keratinocyte layer to the basement membrane in epithelium (4).

The p63 is commonly expressed in stratified squamous epithelium and has been used as a marker for squamous epithelium. Expression of p63 is also a common feature of squamous carcinoma $(5,6)$. High expression of p63 was reported in nasopharyngeal carcinoma (NPC). NPC is a common disease among Southern Chinese but rare in Western population (7). The major histopathological subtypes of nasopharyngeal carcinoma among Southern Chinese are mainly undifferentiated or poorly differentiated carcinoma. Etiological factors including genetic susceptibility, diets and infection of Epstein-Barr virus are believed to be involved in the development of nasopharyngeal carcinoma. Similar to other human cancers, multiple genetic events are also believed to be involved. Identification of early events involved in the development of nasopharyngeal carcinoma may facilitate early detection and diagnosis of this disease.

The histological property of the nasopharyngeal epithelium is more complex than other regions in head and neck. The nasopharynx is a part of the upper respiratory tract at the posterior part of the nasal passageway. The epithelium of the nasopharynx is composed of both pseudostratified columnar ciliated epithelium and stratified squamous epithelium. In adult nasopharynx, the parts near the choanae and adjacent 
roof of nasopharynx are covered by pseudostratified columnar ciliated epithelium, while the lower and posterior regions of the nasopharynx are lined by stratified squamous epithelium. A previous study has shown that $\mathrm{p} 63$ is expressed at the basal and suprabasal layers in the stratified squamous epithelium of nasopharynx, containing the proliferative and presumptive stem cells for the regeneration of the nasopharyngeal epithelium (8). In contrast to most human cancers, p53 mutation is rare in nasopharyngeal carcinoma (9). The $\Delta \mathrm{Np} 63$ is the major isoform of p63 expressed in nasopharyngeal carcinoma. High expression of $\Delta \mathrm{Np} 63$ in nasopharyngeal carcinoma may interfere with the function of the p53 gene and contribute to pathogenesis of nasopharyngeal carcinoma. At present, regulation of p63 expression in nasopharyngeal epithelium is unknown.

Our laboratory has been involved in the study of critical events involved in immortalization and malignant transformation of nasopharyngeal epithelial cells. Somatic cells have limited life span while cancer cells are immortal. Hence, immortalization may represent an early and pre-requisite step in carcinogenesis. Immortalized epithelium may harbor precancerous changes. Identification of these changes may contribute to the understanding of early events involved in carcinogenesis. Our laboratory has established and characterized multiple immortalized nasopharyngeal epithelial cell lines using various immortalization agents including SV40T, HPV16E6E7 and recently telomerase (hTERT) $(10,11)$. These immortalized nasopharyngeal epithelial cell systems have been previously used to define events involved in the pathogenesis of nasopharyngeal carcinoma $(12,13)$. Expression of p63 and its regulation in immortalized nasopharyngeal epithelial cells have not been previously examined. In this study, we have defined the expression profile of various p63 isoforms in primary and immortalized nasopharyngeal epithelial cells and examined some of the factors regulating its expression.

\section{Materials and methods}

Cell culture and cell lines. Both immortalized and malignant nasopharyngeal epithelial cell lines were used in this investigation. Immortalized and malignant epithelial cells from other epithelial tissues including esophageal and ovarian epithelium were also used. Primary nasopharyngeal epithelial cells were established by explanting small size biopsies to collagen coated culture dish. They were immortalized by expression of SV40T, HPV16E6E7 and/or human telomerase (hTERT). Details of the culture methods and immortalization procedures of nasopharyngeal epithelial cells have been previously described (10). The properties of these immortalized nasopharyngeal epithelial cells have been previously reported $(10,11)$. The primary and immortalized nasopharyngeal cells were cultured using 1:1 ratio of Defined Keratinocyte Serum Free Medium supplemented with growth factors (Gibco) and EpiLife ${ }^{\mathrm{TM}}$ medium supplemented with growth factors (Cascade Biologics) and cultured in a $37^{\circ} \mathrm{C}$ incubator with $5 \% \mathrm{CO}_{2}$. Various cancer cells (CNE1, CNE2, HK1, HNE1, HONE1, SUNE1, TW01 and TW04) previously reported to be derived from nasopharyngeal cancer were also included in this study. They were cultured using the RPMI-1640 medium (Sigma) supplemented with 10\% fetal bovine serum (Gibco), $100 \mathrm{U} / \mathrm{ml}$ penicillin and $100 \mu \mathrm{g} / \mathrm{ml}$ streptomycin. Primary and immortalized esophageal cells were cultured in Defined Keratinocyte Serum Free Medium supplemented with growth factors (Gibco) (14). The human ovarian mesothelial cells (HPMC), human ovarian surface epithelial (HOSE) cells and ovarian carcinoma (OVCA) cells were cultured using 1:1 ratio of MCDB105 (Sigma) and M199 (Sigma) medium supplemented with $10 \%$ fetal bovine serum. The properties and culture methods for immortalized HOSE cells have been published (15). HEK293 was cultured in Dulbecco's modified Eagle's (DME) medium HEPES Modification (Sigma) with $10 \%$ fetal bovine serum.

RNA isolation and semi-quantitative reverse-transcriptase $(R T)-P C R$. Total RNA was extracted from the culturing cells at their exponential growth phase using the TRIzol reagent (Invitrogen) according to the manufacturer's protocol. The integrity of the isolated total RNA was confirmed by gel electrophoresis with the presence of discrete $28 \mathrm{~S}$ and $18 \mathrm{~S}$ ribosomal RNA bands in each RNA preparation. Total RNA $(5 \mu \mathrm{g})$ was reversely transcribed to cDNA using the SuperScript II (Invitrogen). The cDNA was then amplified with the $\Delta$ Np63 primers (sense: 5'-CAGACTCAATTTA GTGAG-3'; antisense: 5'-AGCTCATGGTTGGGGCAC-3') (16) and TAp63 primers (sense: 5'-GTCCCAGAGCACA CAGACAA-3'; antisense: 5'-GAGGAGCCGTTCTGAAT CTG-3') (17). The PCR conditions for $\triangle \mathrm{Np} 63$ were as follows: $94^{\circ} \mathrm{C} 2 \mathrm{~min} ; 94^{\circ} \mathrm{C} 30 \mathrm{sec}, 56^{\circ} \mathrm{C} 30 \mathrm{sec}$ and $72^{\circ} \mathrm{C}$ $1 \mathrm{~min}$ for 30 cycles; $72^{\circ} \mathrm{C} 7 \mathrm{~min}$. TAp63 was amplified using the followings PCR conditions: $94^{\circ} \mathrm{C} 2 \mathrm{~min} ; 94^{\circ} \mathrm{C}$ $30 \mathrm{sec}, 54^{\circ} \mathrm{C} 40 \mathrm{sec}$ and $72^{\circ} \mathrm{C} 30 \mathrm{sec}$ for 30 cycles; $72^{\circ} \mathrm{C}$ 7 min. p63 $\alpha, \beta$ and $\gamma$ were amplified using the primers sequences from Di Como et al (17). PCR conditions for Cterminal isoforms were: $94^{\circ} \mathrm{C} 2 \mathrm{~min} ; 94^{\circ} \mathrm{C} 30 \mathrm{sec}, 57^{\circ} \mathrm{C}$ $40 \mathrm{sec}$ and $72^{\circ} \mathrm{C} 30 \mathrm{sec}$ for 2 cycles; $94^{\circ} \mathrm{C} 30 \mathrm{sec}, 55^{\circ} \mathrm{C} 40 \mathrm{sec}$ and $72^{\circ} \mathrm{C} 30 \mathrm{sec}$ for 28 cycles; $72^{\circ} \mathrm{C} 7 \mathrm{~min}$. RT-PCR for $\beta$ actin transcript was used as the internal PCR control using the specific primer sequences (sense: 5'-CCTGGCACC CAGCACAAT-3'; antisense: 5'-GGGCCGGACTCGTCA TAC-3'). The PCR conditions for $B$-actin were: $94^{\circ} \mathrm{C} 2 \mathrm{~min}$; $94^{\circ} \mathrm{C} 25 \mathrm{sec}, 56^{\circ} \mathrm{C} 25 \mathrm{sec}$ and $72^{\circ} \mathrm{C} 30 \mathrm{sec}$ for 20 cycles; $72^{\circ} \mathrm{C} 7 \mathrm{~min}$.

Reverse transcriptase quantitative PCR (RT-QPCR). The expression of the $\triangle \mathrm{Np} 63$ gene in various cell types was first examined by semi-quantitative RT-PCR. Accurate determination of expression levels of the mRNA was then achieved by quantitative RT-PCR (BioRad). The $\triangle \mathrm{Np} 63$ transcript was quantified by Q-PCR using the $\mathrm{iCycler}^{\mathrm{iQ}} \mathrm{T}^{\mathrm{TM}}$ (BioRad) with SYBR-Green (Molecular Probe). In brief, $1 \mu 1$ of cDNA was added in a $24 \mu 1$ reaction mixture containing 0.5X SYBR-Green, Gibco PCR buffer (Invitrogen), $0.6 \mu \mathrm{M}$ $\mathrm{MgCl}_{2}, 0.4 \mu \mathrm{M}$ dNTP, $0.5 \mu \mathrm{M}$ primer sets and $0.5 \mathrm{U}$ Gibco Taq Polymerase (Invitrogen). Q-PCR were performed in triplicates and repeated three times. The specificity of the PCR product was confirmed by the melt-curve analysis (BioRad) and by $2 \%$ agarose gel electrophoresis revealing a 
single PCR product with expected size. The expression level of $\Delta \mathrm{Np} 63$ transcript was internally normalized using the expression level of $\beta$-actin. Expression level of $\Delta \mathrm{Np} 63$ transcript in nasopharyngeal epithelial cells was calculated as $2^{-\Delta \Delta C t}$, where the $\Delta C_{t}$ represents the difference between thresholds of candidate gene and $\beta$-actin $\left(\mathrm{C}_{t \Delta N p 63}-\mathrm{C}_{t \beta \text {-actin }}\right)$ and the $\Delta \Delta C_{t}$ means the difference between the $\Delta C_{t}$ of nasopharyngeal epithelial cells and primary nasopharyngeal cells.

Western blot analysis. The expression of p63 was examined by Western blot analysis. Details of the procedures have been previously published (12). The cell pellets harvested were washed once with phosphate buffered saline and lysed with the lysis buffer [150 mM NaCl, $50 \mathrm{mM}$ Tris- $\mathrm{HCl}$ (pH 8.0), $1 \%$ Nonidet P40, $5 \mathrm{mg} / \mathrm{ml}$ sodium deoxycholate, $1 \mathrm{mg} / \mathrm{ml}$ sodium dodecyl sulfate, $1 \mathrm{mM}$ PMSF, $2.5 \mathrm{ng} / \mu 1$ leupeptin, $1 \mathrm{ng} / \mu \mathrm{l}$ aprotinin]. Total protein $(15 \mu \mathrm{g})$ was loaded and separated on sodium dodecyl sulfate polyacrylamide gel electrophoresis. The gel was then transferred onto a PVDF membrane (Amersham). The antibodies and the dilutions used in this study are: p63 (p63 Ab-3; 1:200; NeoMarkers), p40 (1:200; Calbiochem), p51B (Ab-1; 1:200; Oncogene), involucrin (Ab-1 Clone SY5; 1:1000; NeoMarkers), actin (1:1000; Santa Cruz Biotechnology), p53 (DO-7; 1:2000; Dako), caspase-3 (1:500; Cell Signaling Technologies), cleaved caspase-3 (1:500; Cell Signaling Technologies), cleaved PARP (1:500; Cell Signaling Technologies), integrin B4 (1:500; Santa Cruz Biotechnology), laminin $\gamma$-2 (1:500; Santa Cruz Biotechnology), EGFR (1:5000; Santa Cruz Biotechnology), Bcl-2 (1:500; Santa Cruz Biotechnology), Bcl-xL (1:1000; Cell Signaling Technologies) and Bax (1:500; Cell Signaling Biotechnology).

Determination of growth rate by $\left[{ }^{3} \mathrm{H}\right]$-thymidine incorporation. The cells were grown on $24-w e l l$ plate. The cells were subjected to treatments by different concentration of all-trans retinoic acid (Sigma) for two days. At the end of the treatment period, $0.5 \mu \mathrm{Ci}\left[{ }^{3} \mathrm{H}\right]$-thymidine diluted by culture medium was added to each well with a dispenser. The plate was kept on top of a tray to prevent leakage and incubated at $37^{\circ} \mathrm{C}$ incubator with $5 \% \mathrm{CO}_{2}$ for 3-4 h. The medium was pipetted away from each well and the cells were washed with $1 \mathrm{ml}$ cold $1 \mathrm{X}$ PBS. The macromolecule was precipitated with $1 \mathrm{ml}$ cold $5 \%(\mathrm{w} / \mathrm{v})$ trichloroacetic acid for several minutes. This step was repeated once. The cells were washed with $1 \mathrm{ml}$ cold absolute methanol. The methanol was discarded and the plate was air-dried in fume hood overnight or until all methanol evaporated. $0.2 \mathrm{~N} \mathrm{NaOH}(500 \mu 1)$ was added to each well using a dispenser. The plate was incubated at $37^{\circ} \mathrm{C}$ incubator with $5 \% \mathrm{CO}_{2}$ for $10 \mathrm{~min}$. The cells were dissolved by pipetting up and down several times with a $1 \mathrm{ml}$ pipette tip. Lysate $(300 \mu 1)$ was transferred to a scintillation vial. Aqueous scintillation fluid of about $3 \mathrm{ml}$ was added to each vial. The vial was mixed by shaking up and down. The sample was ready for scintillation counting.

Induction of differentiation in immortalized nasopharyngeal epithelial cells. The immortalized nasopharyngeal epithelial cell system provides us a unique opportunity to examine the relationship between differentiation and expression of
$\Delta$ Np63. The immortalized nasopharyngeal epithelial cells were subjected to treatment by different concentration of different differentiating agents, including serum, dialysed serum, calcium chloride (Sigma), human recombinant $\mathrm{TNF} \alpha$ (Gibco), human transforming growth factor $\beta 1$ (TGFß1) (Calbiochem) and all-trans retinoic acid (Sigma). The successful induction of differentiation of immortalized nasopharyngeal epithelial cells was monitored by the expression of involucrin, which is a marker for squamous differentiation.

Induction of apoptosis in immortalized nasopharyngeal epithelial cells. To study the p63 status in immortalized nasopharyngeal epithelial cells during apoptosis, the cells were treated by $1 \mu \mathrm{M}$ staurosporin (Calbiochem) to induce apoptosis. The treated cells were harvested at different time $(0,1.5,3$ and $6 \mathrm{~h})$. Successful induction of apoptosis in immortalized nasopharyngeal epithelial cells was checked by the expression of cleaved caspase- 3 and cleaved PARP.

Loss of function analysis of $\triangle N p 63 \alpha$ in immortalized nasopharyngeal epithelial cells. siRNA against $\Delta \mathrm{Np} 63 \alpha$ was designed according to the known $\Delta \mathrm{Np} 63 \alpha$ sequence. siRNA against GFP (siGFP) and $\triangle \mathrm{Np} 63 \alpha$ stealth siRNA duplex oligoribonucleotides 5'-CCGCAAUAAGCAACAGCGC AUCAAA-3' (Invitrogen) were used in this study. The siGFP was used as the control. The stealth siRNA were transfected to target cells using lipofectamin 2000 (Invitrogen) according to manufacturer's instruction with modification. Cells at $70 \%$ confluent are ready for transfection. For cells growing on $25-\mathrm{cm}^{2}$ flask, medium were replaced with $2.5 \mathrm{ml}$ antibioticfree medium. siRNA of $400 \mathrm{pmol}$ was diluted by adding to $250 \mu 1$ antibiotic-free medium. Lipofectamin 2000 of $6 \mu 1$ per 100 pmol siRNA was added to another $250 \mu 1$ antibiotic-free medium. The tube with lipofectamin in medium was incubated at room temperature for $5 \mathrm{~min}$. Then the diluted siRNA was mixed with the diluted lipofectamin. The mixture was incubated at room temperature for $15 \mathrm{~min}$. The siRNAlipofectamin mixture was added to the flask of target cells and incubated at $37^{\circ} \mathrm{C}$ incubator with $5 \% \mathrm{CO}_{2}$ for $6-8 \mathrm{~h}$. The medium was changed. Cells were harvested 2 days after transfection.

Generation of retroviral construct and retrovirus infection. The full length $\triangle \mathrm{Np} 63 \alpha$ gene was PCR amplified from cDNA of the nasopharyngeal carcinoma cell line, HK1. 5'-GAAGT TATCAGTCGACATGTTGTACCTGGAAAAC-3' (sense) and 5'-ATGGTCTAGAAAGCTTTCACTCCCCCTCCT CTTT-3' (antisense) were the primers sequences used. PCR conditions were as follows: $94^{\circ} \mathrm{C} 2 \mathrm{~min} ; 94^{\circ} \mathrm{C} 30 \mathrm{sec}, 59^{\circ} \mathrm{C}$ $30 \mathrm{sec}$ and $72^{\circ} \mathrm{C} 2 \mathrm{~min} 30 \mathrm{sec}$ for 30 cycles; $72^{\circ} \mathrm{C} 7 \mathrm{~min}$. The PCR products were separated in $1.5 \%$ agarose gel and the product size of around $1800 \mathrm{bp}$ represents the target product. This PCR amplified product was excised from the gel. The product was cloned into linearized pDNR-Dual donor vector using the BD in-fusion PCR cloning kit (BD Biosciences Clontech). Correct sequence of the $\Delta \mathrm{Np} 63 \alpha$ insert was confirmed by sequencing. The pDNR-Dual- $\Delta$ Np63 $\alpha$ generated was then subcloned into acceptor vector, $\mathrm{pLp}$-LNCX, using the BD creator DNA cloning kit (BD Biosciences Clontech). The pLp-LNCX- $\triangle \mathrm{Np} 63 \alpha$ vector was cotransfected with 
pVSV-G (kindly provided by Dr H.L. Chen, Department of Microbiology, Li Ka Shing Faculty of Medicine, University of Hong Kong, Hong Kong) into the Phoenix 293 packaging cell line using Fugene 6 transfection reagent (Roche) according to the manufacturer's instruction. The medium was replaced with fresh medium on the next day. Two days after transfection, the supernatant containing the retrovirus was collected and filtered through $0.45 \mu \mathrm{m}$ filter to get rid of any floating cells. The primary cells to be infected were replaced with $4 \mathrm{ml}$ fresh medium. A final concentration of $4 \mu \mathrm{g} / \mathrm{ml}$ Polybrene (Sigma) and $1 \mathrm{ml}$ of the supernatant were added to the cells. The cells were incubated in a $37^{\circ} \mathrm{C}$ incubator with $5 \% \mathrm{CO}_{2}$ for about $16 \mathrm{~h}$. Medium were replaced after incubation. The cells were selected with G418 for 2 weeks.

\section{Results}

The expression of p63 protein was downregulated in senescent and differentiating primary nasopharyngeal epithelial cells but is elevated in immortalized nasopharyngeal epithelial cells. We first examined the expression of p63 in three primary nasopharyngeal epithelial cells (NP460, NP446 and NP550) and compared their expression levels with a telomerase immortalized nasopharyngeal epithelial cells (NP460hTERT) and an established nasopharyngeal carcinoma cell line (HK1) by Western blotting (Fig. 1A). Various antibodies were used to detect p63 and its various isoforms. The p63 antibody detects both the $\Delta \mathrm{Np} 63$ and TAp63 isoforms of the p63 and has been commonly used in other studies to detect expression of p63 in squamous carcinoma both in Western blotting and immunohistochemistry. The p40 detects the expression of $\Delta \mathrm{Np} 63$, while p51B detects the expression of TAp63. As seen in Fig. 1A, the expression of p63 was detected at high levels in the NPC cell line (HK1), the telomerase immortalized nasopharyngeal epithelial cells (NP460hTERT) and primary nasopharyngeal epithelial cells (NP446 and NP550) at their early stages of population doubling (PD 7 and 6 respectively) when they were still at their proliferative stages. Expression of p63 decreased in primary nasopharyngeal cells at late passages when the cells slowed down in proliferation rate and entered senescence. In NP460 (PD 15) and NP550 (PD 23), the senescent cells expressed terminal differentiation properties, which was confirmed by the expression of involucrin, a cross-linking protein of the cornified envelope of epidermis commonly used as marker for terminal differentiation (18). In general, an inverse relationship between the expression levels of involucrin and p63 was observed in both primary and immortalized nasopharyngeal epithelial cells (Fig. 1B). The NP460 at PD 13 (entering senescence) expressed low level of p63 but high level of involucrin, while increased expression of p63 and decreased expression of involucrin were observed during immortalization and proliferative culture (NP460hTERT). Hence, it can be concluded that expression of p63 is suppressed in senescent and terminal differentiated nasopharyngeal epithelial cells. In contrast, the telomerse-immortalized nasopharyngeal epithelial cells are proliferative and with elevated expression of p63 but low expression of involucrin.
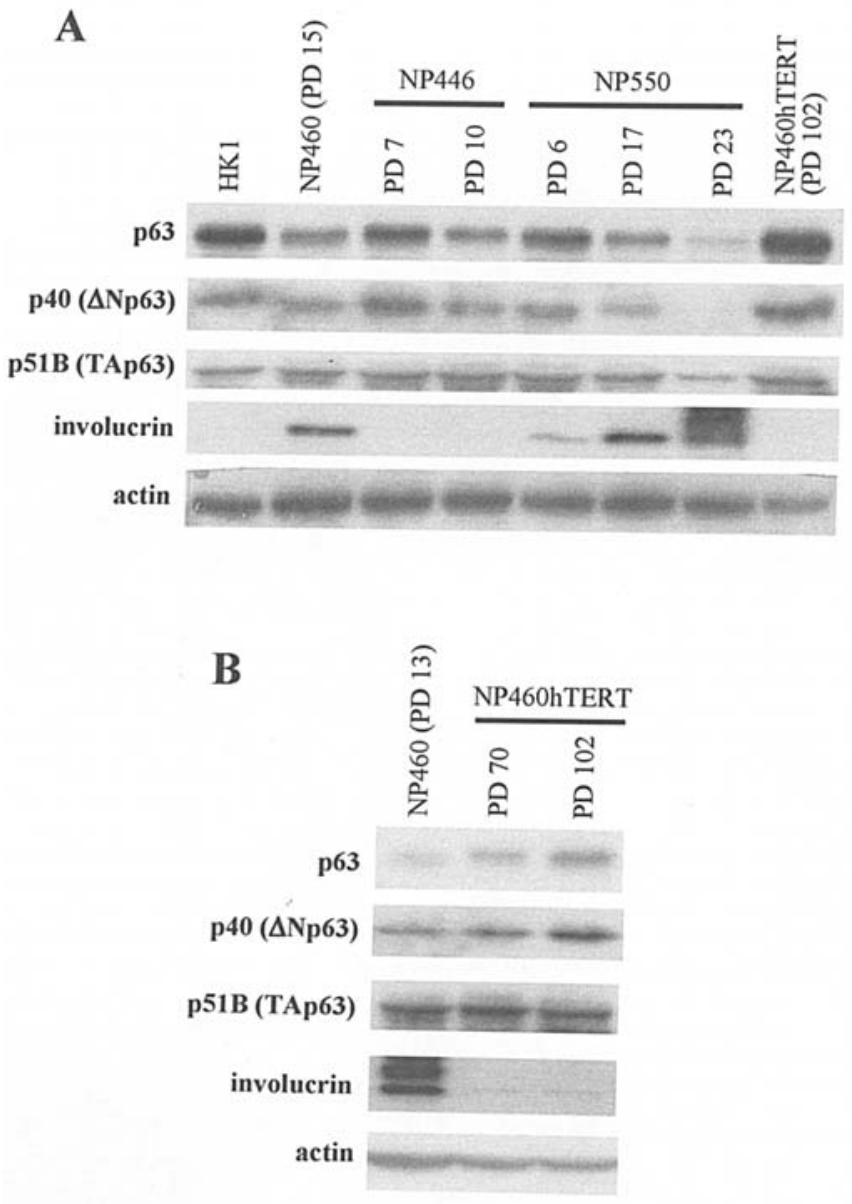

Figure 1. Expression of p63 protein in epithelial cells. (A) Western blotting showing p63 expression in nasopharyngeal cells at different cell stages of immortalization. P63 expression was higher in immortalized cells (NP460hTERT) than the cells derived from primary culture (NP460, NP446 and NP550). Expression of p63 decreased when the primary nasopharyngeal epithelial cells approach senescence or differentiation. (B) Expression of p63 increased during immortalization of nasopharyngeal epithelial cells. Cells derived from primary culture, the NP460 cells, was highly differentiated as indicated by the expression of the differentiation marker, involucrin. The increase in expression of p63 during immortalization was correlated with decrease expression of involucrin.

By comparing the expression levels of p63 and its two isoforms $\Delta \mathrm{Np} 63$ and TAp63 using specific antibodies, $\mathrm{p} 40$ and $\mathrm{p} 51 \mathrm{~B}$ respectively, it can be concluded that suppression of p63 expression is mainly related to the downregulation of $\Delta \mathrm{Np} 63$ but not TAp63. The TAp63 isoform remained fairly constant in primary, immortalized and malignant nasopharyngeal epithelial cells, in both proliferative and senescent stages (Fig. 1).

The $\triangle N p 63 \alpha$ mRNA was the predominant p63 transcript expressed in primary nasopharyngeal epithelial cells and nasopharyngeal carcinoma cells. To identify the specific isoform of p63 expressed in nasopharyngeal epithelial cells, we examined the level of transcript of various isoforms of p63 by semi-quantitative RT-PCR. The RT-PCR analysis was consistent with the Western blotting in that $\Delta \mathrm{Np} 63$ was the major transcript detected in primary nasopharyngeal 

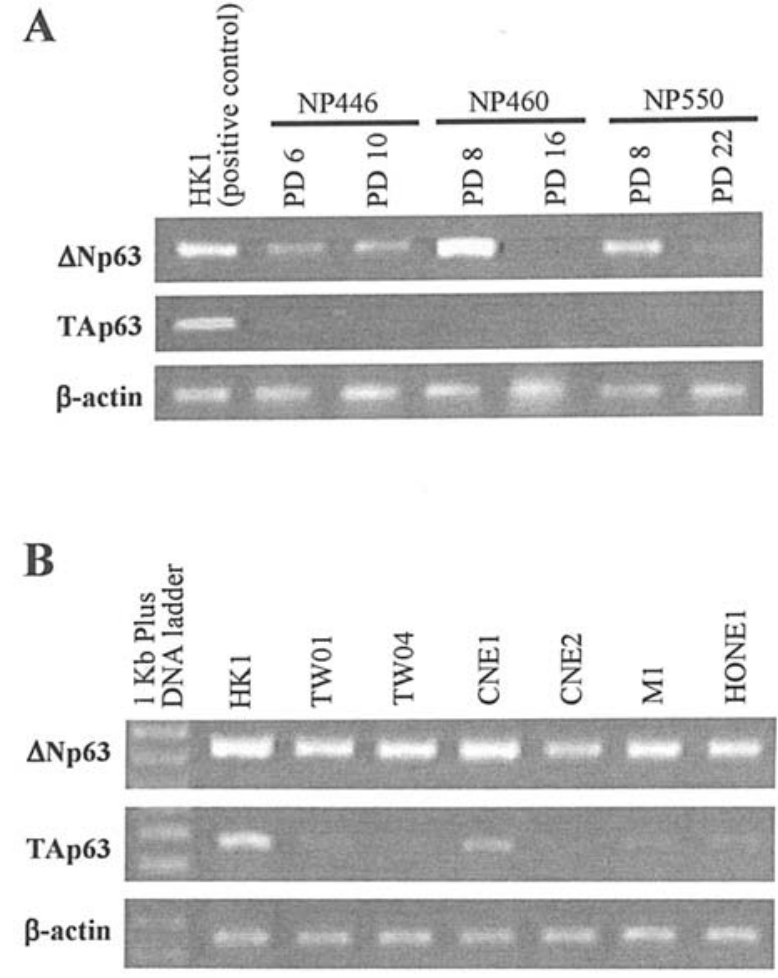

Figure 2. Expression of different N-terminal p63 mRNA in nasopharyngeal epithelial cells. (A) Nasopharyngeal epithelial cells derived from primary cells. (B) Nasopharyngeal carcinoma cells. Both $\triangle$ Np63 and TAp63 mRNA were detected in all the nasopharyngeal epithelial cells examined. The levels of $\Delta \mathrm{Np} 63$ transcripts were higher than that of TAp63.

epithelial cells (NP446, NP460 and NP550) (Fig. 2A). TAp63 transcript was low and undetectable in all primary nasopharyngeal epithelial cells examined. Similar to the Western blotting results of p63, the mRNA level of $\Delta \mathrm{Np} 63$ was low in primary nasopharyngeal epithelial cells undergoing senescence (NP460 at PD 16 and NP550 at PD 22) (Fig. 2A). In established cancer cells, the $\Delta \mathrm{Np} 63$ was also the major p63 isoform detected, except for HK1 where high levels of both $\Delta \mathrm{Np} 63$ and TAp63 were detected (Fig. 2B). The difference in the expression level of p63 isoforms in HK1 may be related to the unique differentiated properties of HK1, which is derived from a well-differentiated squamous carcinoma of the nasopharyngeal region (19). Other nasopharyngeal carcinoma cell lines used were reported to be derived from poorly-differentiated carcinoma (TW01, TW04, CNE1, CNE2, M1 and HONE1) which is a common histological type of nasopharyngeal carcinoma among Southern Chinese (Fig. 2B).

Furthermore, we examined the levels of various Cterminal isoforms $(\alpha, \beta$ and $\gamma)$ of $\mathrm{p} 63$ by semi-quantitative RT-PCR. The p63 $\alpha$ transcript was detected at a much higher level compared to the $\beta$ and $\gamma$ transcripts in primary nasopharyngeal epithelial cells (Fig. 3A). In nasopharyngeal carcinoma cell lines, p63 $\alpha$ transcript was also the dominant transcript detected (Fig. 3B). Interestingly, high levels of $\beta$ and $\gamma$ transcripts of p63 were also detected in HK1 but not in other nasopharyngeal carcinoma cell lines, which may be a characteristic of well-differentiated squamous carcinoma.
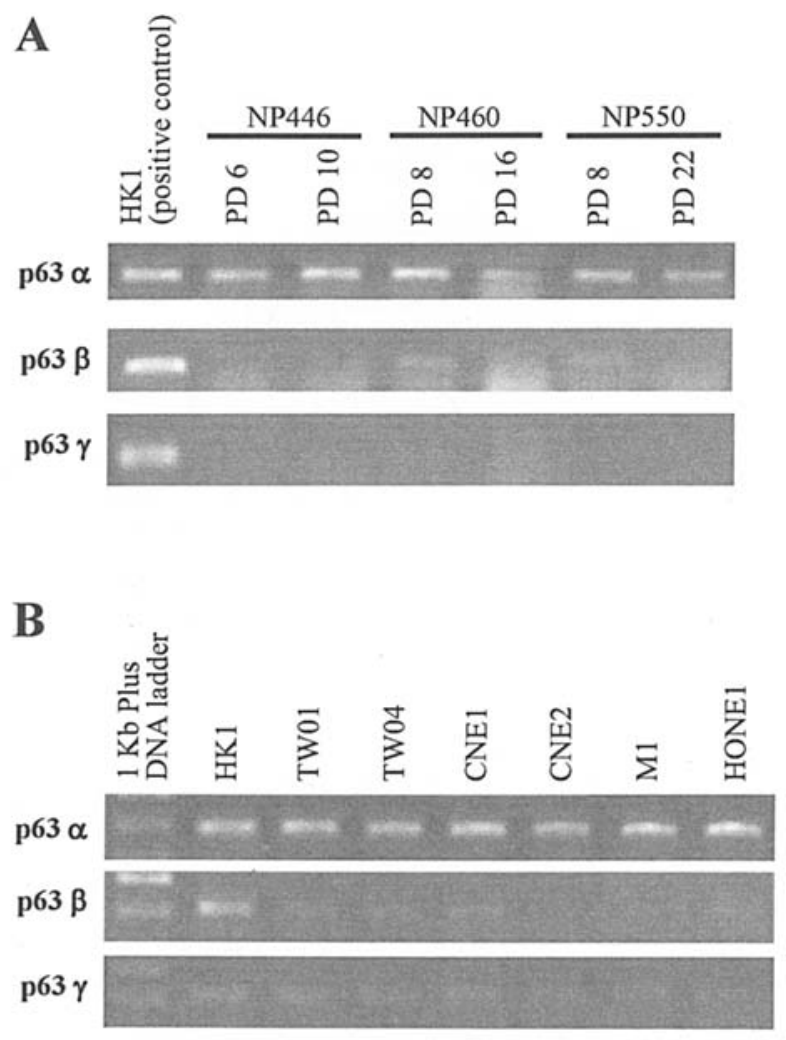

Figure 3. Expression of different C-terminal p63 mRNA in nasopharyngeal epithelial cells. (A) Nasopharyngeal epithelial cells derived from primary cultures. (B) Nasopharyngeal carcinoma cells. All the three C-terminal p63 isoforms $(\alpha, \beta$ and $\gamma)$ were detected in all the nasopharyngeal epithelial cells examined. The levels of p63 $\alpha$ transcripts were significantly higher than the levels of other two isoforms.

Quantitative real-time PCR confirmed elevated expression of $\triangle N p 63$ in immortalized nasopharyngeal epithelial cells and nasopharyngeal carcinoma cells. The expression levels of $\triangle \mathrm{Np} 63$ in the primary nasopharyngeal epithelial cells (NP550 and NP460), immortalized nasopharyngeal epithelial cells (NP460hTERT) at early and late passages (PD 28 and PD 72 respectively), and several previously reported nasopharyngeal carcinoma cells (SUNE1, CNE2, M1, HONE1, TW04, HNE1 and HK1) were compared (Fig. 4). The expression of $\Delta \mathrm{Np} 63$ in the primary nasopharyngeal epithelial cells before immortalization was low compared to immortalized nasopharyngeal epithelial cells. The primary nasopharyngeal epithelial cells had a lower proliferation rate compared to immortalized nasopharyngeal epithelial cells and nasopharyngeal carcinoma cells. Interestingly, there was a mark increase (about 32-fold) in expression level of $\Delta \mathrm{Np} 63$ observed at late passage of the telomerase-immortalized nasopharyngeal epithelial cells (NP460hTERT at PD 72), which is indicative of an association between $\triangle \mathrm{Np} 63$ and proliferative capacity of immortalized nasopharyngeal epithelial cells.

Restricted expression of $\triangle N p 63$ to epidermal cells from ectodermal and endodermal origins. We then examined the expression of $\Delta \mathrm{Np} 63$ in immortalized and cancer cell 


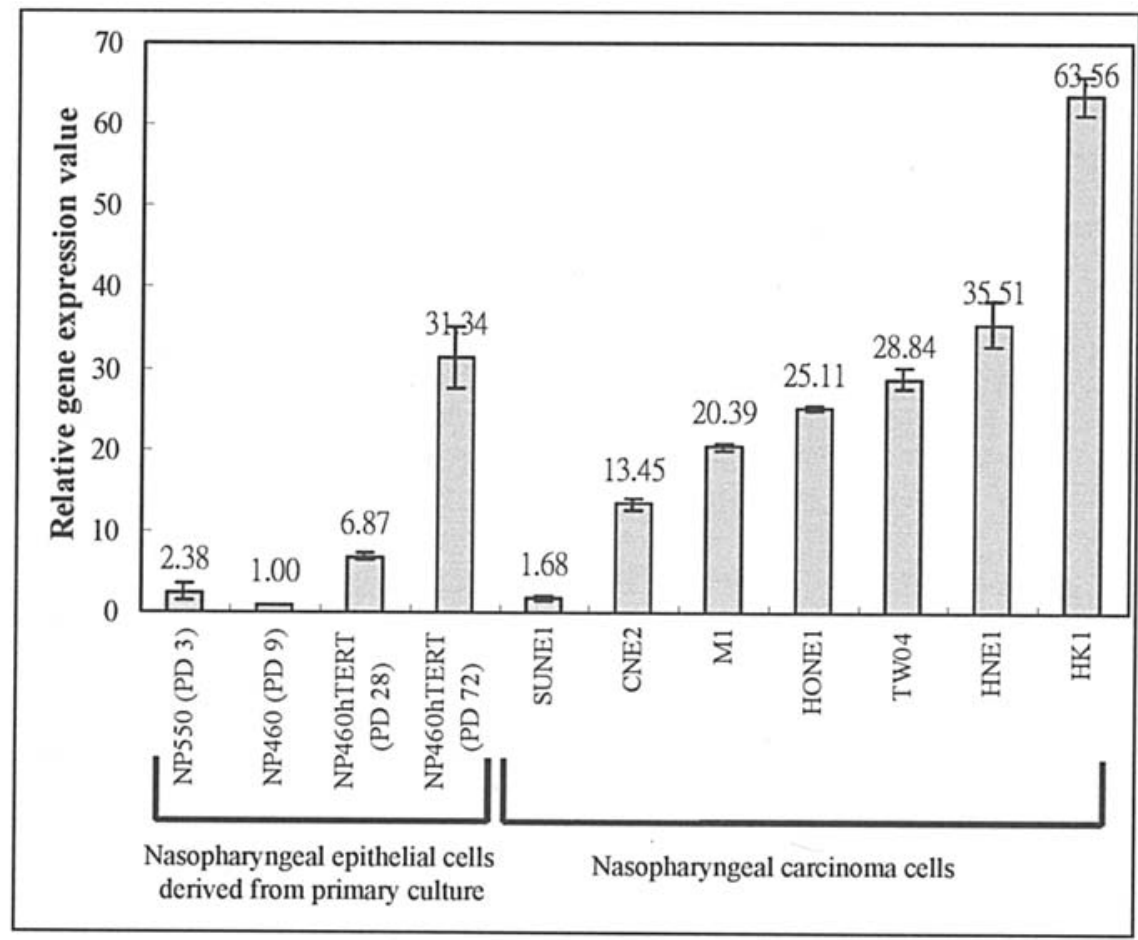

Figure 4. Quantitative real-time PCR of $\Delta \mathrm{Np} 63$ gene in nasopharyngeal cells. All the nasopharyngeal cells used have $\Delta \mathrm{Np} 63 \mathrm{mRNA}$ expression. The lowest expression was detected in the primary cells, and the highest in the NPC cell line, HK1. The NPC cell lines have a higher level of the $\Delta$ Np63 than the immortalized cells at their early stage of immortalization (NP460hTERT at PD 28). The expression level of $\Delta$ Np63 detected was higher at the later stage of immortalization (NP460hTERT at PD 72) and was comparable to that in nasopharyngeal carcinoma cells.

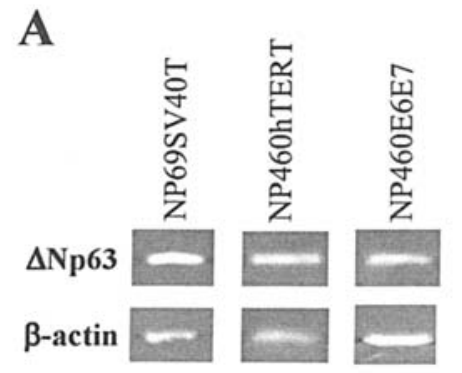

B

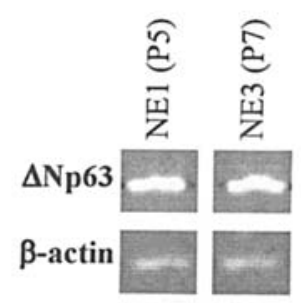

C

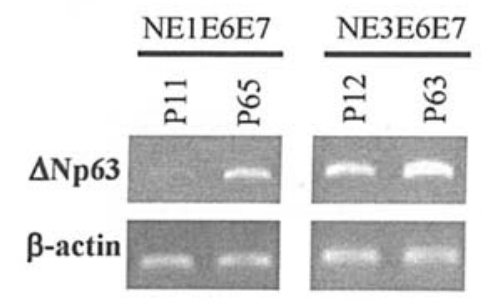

Figure 5. Expression of $\triangle \mathrm{Np} 63 \mathrm{mRNA}$ in immortalized nasopharyngeal epithelial cells and different esophageal cells. (A) Immortalized nasopharyngeal epithelial cells. (B) Cells derived from primary culture of esophageal cells. (C) Immortalized esophageal cells. Expression of $\triangle \mathrm{Np} 63 \mathrm{mRNA}$ was detected in the nasopharyngeal and esophageal cells, which are from the ectodermal and endodermal origins respectively. In the immortalized esophageal cells, the expression level of $\Delta \mathrm{Np} 63$ detected was higher at late passages.

lines from different developmental lineages by RT-PCR. The $\Delta \mathrm{Np} 63$ transcript could be detected in immortalized nasopharyngeal epithelial cells regardless of the immortalizing agents used (SV40T, hTERT and HPV16E6E7) (Fig. 5A). The $\Delta \mathrm{Np} 63$ could also be detected in both primary esophageal epithelial cells (NE1 and NE3) (Fig. 5B) and esophageal epithelial cells immortalized by HPV16E6E7 (NE1E6E7 and NE3E6E7) (Fig. 5C). In contrast, $\Delta \mathrm{Np63}$ transcript was not detected in human peritoneal mesothelial cells (HPMC), human ovarian surface epithelial (HOSE) cells and ovarian carcinoma (OVCA) cells (Fig. 6). The nasopharyngeal and esophageal epithelial cells are derived from ectodermal and endodermal lineages respectively, while the peritoneal and ovarian epithelial cells were from mesodermal lineage. Cells from both ectodermal and endodermal lineages could undergo squamous differentiation but those from mesodermal origin could not. It could be concluded at this stage that expression of $\Delta \mathrm{Np} 63$ is restricted to cells derived from ectodermal and endodermal origins but not from mesodermal origin.

Expression of p63 was downregulated in immortalized nasopharyngeal epithelial cells upon induction of terminal differentiation. Primary keratinocytes are prone to undergo terminal differentiation when exposed to serum and high calcium in culture. We successfully induced differentiation in an immortalized nasopharyngeal epithelial cell line (NP460hTERT) by treatment with $10 \%$ fetal bovine serum (FBS) (Fig. 7A). Successful induction of terminal differentiation in NP460hTERT was confirmed by the expression of involucrin, which is a spinous marker of differentiated 

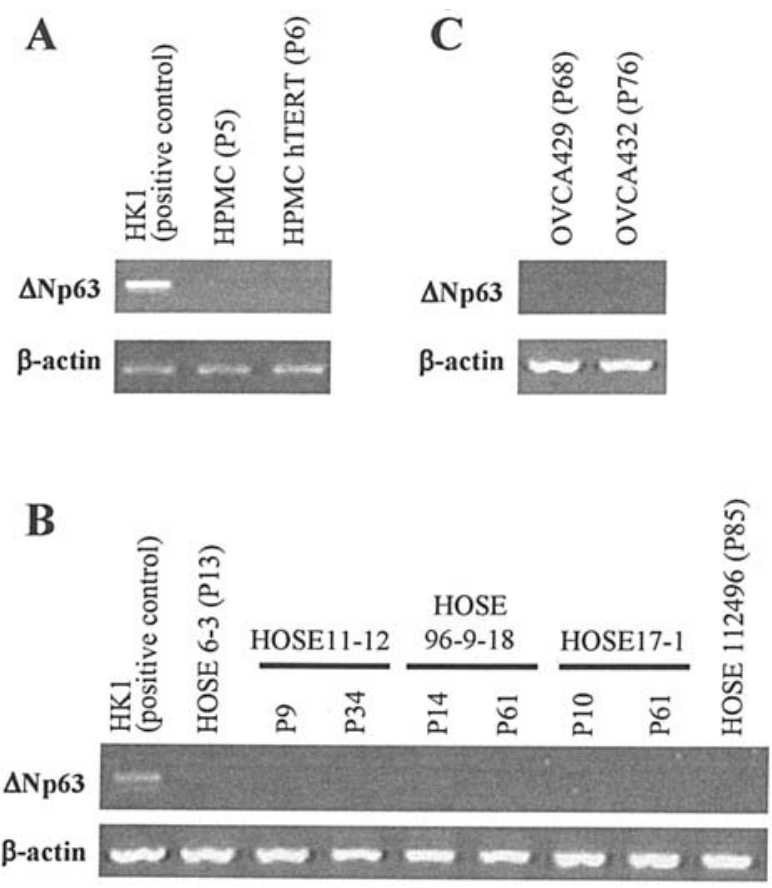

Figure 6. Expression of $\triangle \mathrm{Np} 63 \mathrm{mRNA}$ in cells derived from peritoneal and ovarian surface epithelial origins. (A) Human peritoneal mesothelial cells. (B) Immortalized human ovarian surface epithelial cells. (C) Ovarian carcinoma cells. There was no $\triangle \mathrm{Np} 63$ mRNA expression detected in these cells which are derived from the mesodermal origin.

keratinocytes (20). Induction of expression of involucrin was also observed in immortalized NP460hTERT cells treated with dialysed serum having low calcium concentration (Fig. 7A). In addition to the expression of involucrin, the serumtreated cells also assumed enlarged and flattened morphology which are characteristics of differentiated cells in culture (Fig. 8). The level of $\Delta \mathrm{Np} 63$ protein expression, which was detected by the p40 antibody, also decreased in the serum-treated cells. In contrast, the TAp63 protein, which was detected by the p51B antibody, did not reveal significant changes after serum treatment (Fig. 7A). The effect of serum on the downregulation of p63 was quite effective and was detected in the presence of $1.25 \%$ dialysed serum (Fig. 7B). Serum treatment could also effectively induce differentiation of NP69SV40T, an immortalized nasopharyngeal epithelial cell line, as indicated by the increased expression of involucrin and lowered expression of p63 (Fig. 7C). This again confirmed our previous observation that nasopharyngeal epithelial cells undergoing terminal differentiation suppress p63 expression. There are reports showing that calcium ion induced terminal differentiation in human epidermal keratinocytes $(21,22)$. The effect of calcium on induction of terminal differentiation in nasopharyngeal epithelial cells is unknown. Our results indicated that calcium has minimal or no change in regulation of involucrin and p63 expressions in immortalized nasopharyngeal epithelial cells (NP460hTERT) (Fig. 7A and B). Apparently, the immortalized NP460hTERT cells are not responsive to calcium treatment with respect to induction of terminal differentiation.

Treatment with TGF $\beta$ led to downregulation of p63 in immortalized nasopharyngeal epithelial cells. TGFß is one of
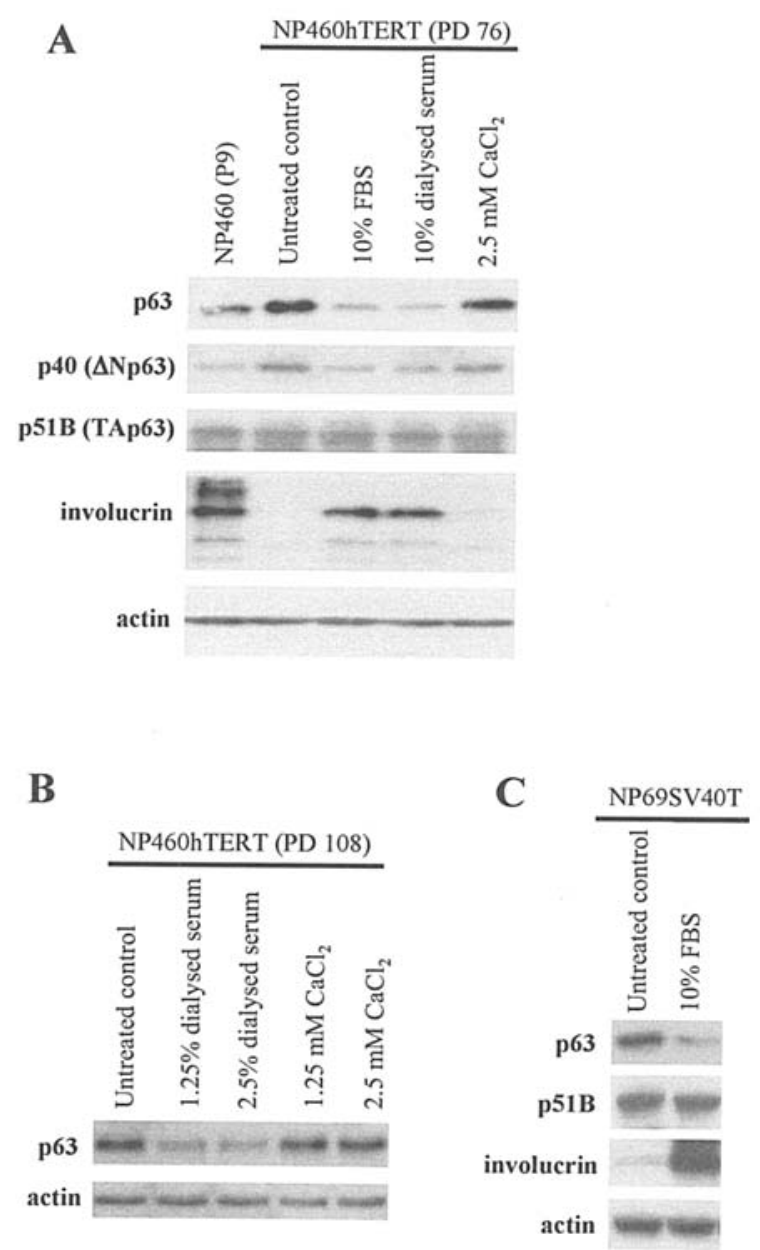

Figure 7. Expression of p63 in immortalized nasopharyngeal cells during differentiation. (A) After treatment with serum (both FBS and dialysed serum), the immortalized nasopharyngeal cells were induced to differentiate as indicated by the expression of involucrin. The differentiated cells expressed a lower level of p63. Treatment with calcium ion did not induce differentiation or change in p63 expression. (B) Treatment with as low as $1.25 \%$ serum decreased expression of p63. (C) Induction of differentiation in SV40 immortalized nasopharyngeal epithelial cells (NP69SV40T) by fetal bovine serum (FBS) effectively suppressed p63 expression.

the differentiation factors present in serum. The effect of TGFß on p63 and involucrin expressions in immortalized nasopharyngeal epithelial cells were examined. Immortalized nasopharyngeal epithelial cells and nasopharyngeal carcinoma cells were treated with $15 \mathrm{ng} / \mathrm{ml}$ TGF 3 for 2 days (Fig. 9). Treatment with TGFß decreased the expression of p63 at early passage of immortalized cells (NP460hTERT at PD 76) but was ineffective for late passage (NP460hTERT at PD 113) (Fig. 9A). The involucrin level was however not significantly altered by TGFß treatment. The exact mechanism involved is not clear at this stage but may be related to the responsiveness of immortalized nasopharyngeal epithelial cells to the growth inhibitory effect of TGFß. Immortalized nasopharyngeal epithelial cells upon prolonged propagation eventually lost their responses to growth inhibition by TGFß (unpublished data) and were not responsive to downregulation of $\mathrm{p} 63$ by TGFß.

Retinoic acid effectively downregulated p63 expression in immortalized nasopharyngeal epithelial cells. The retinoic 

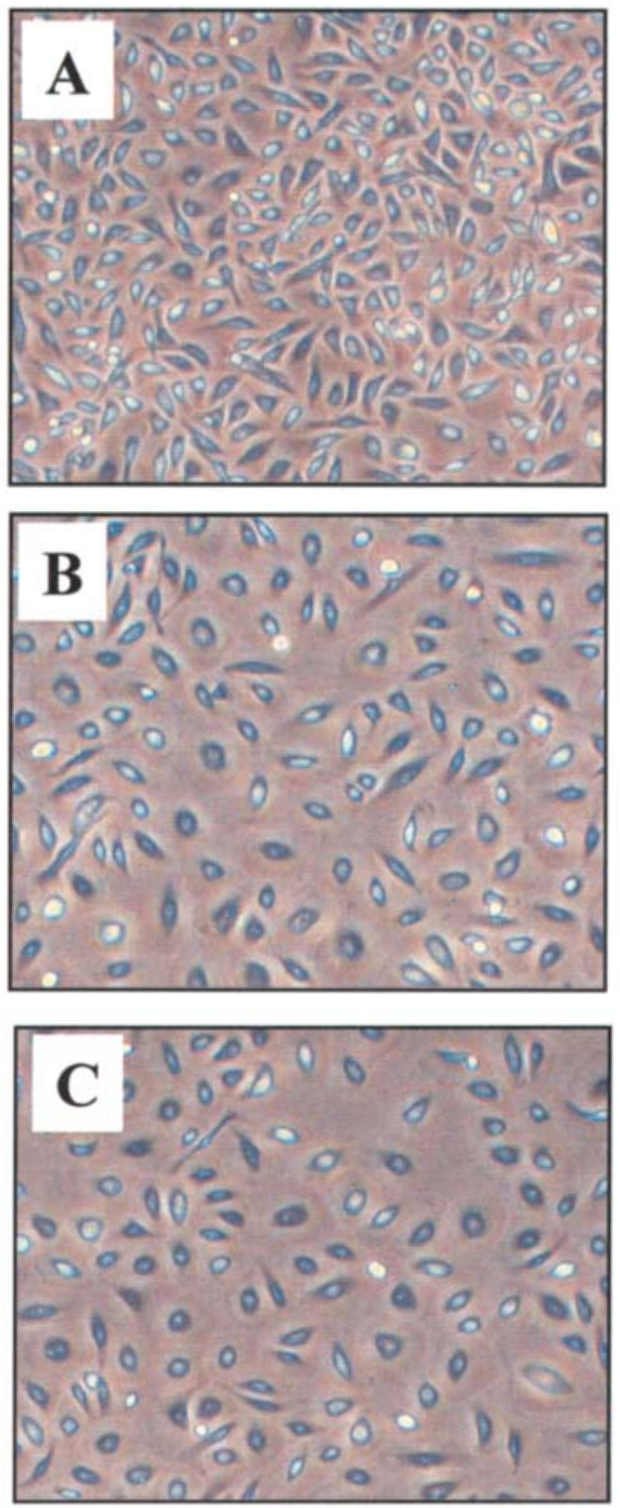

Figure 8. The morphology of immortalized nasopharyngeal epithelial cells (NP460hTERT) treated with serum or dialysed serum. (A) Untreated control. (B) Treated with 10\% FBS. (C) Treated with $10 \%$ dialysed serum. The serum treated cells assumed an enlarged and flattened morphology similar to differentiated cells in culture.

acid is a powerful agent in modulating differentiation status in respiratory epithelium. Our result showed that treatment with all-trans retinoic acid (ATRA) could effectively lower p63 expression in a dose-dependent manner in NP460hTERT cells (Fig. 10A). Inhibitory effect of ATRA on p63 expression was also observed in NP69SV40T (a nasopharyngeal epithelial cell line immortalized by SV40T), SCC12F (a head and neck squamous carcinoma cell line) and $\mathrm{HaCaT}$ (a spontaneously immortalized line of human foreskin keratinocyte) (Fig. 10B). In general, ATRA inhibits terminal differentiation of squamous epithelium and supports secretary differentiation of respiratory epithelium. Inhibition of involucrin expression by ATRA was observed in the immortalized NP460hTERT cells (Fig. 10C). In NPC cells examined, however, involucrin expression was low and ATRA treatment has insignificant effects on its expression (Fig. 10C). Yet, the effect of reduction in p63 by ATRA could still be observed in most of
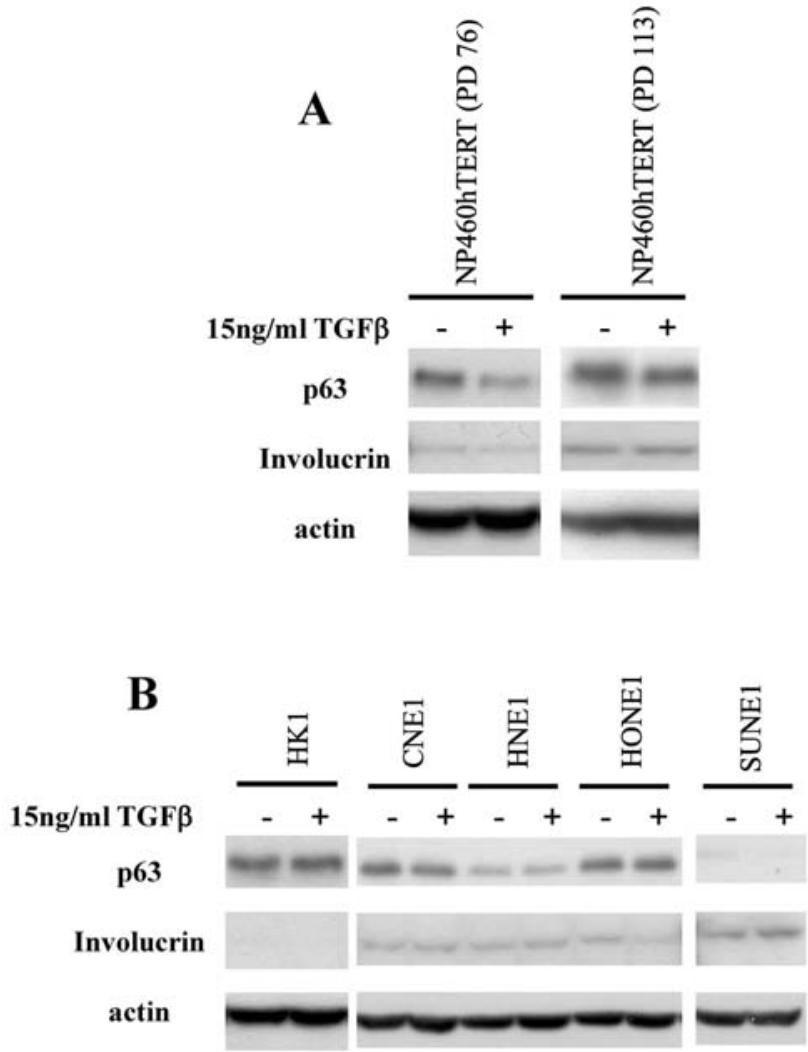

Figure 9. Expression of p63 in nasopharyngeal cells treated with TGFß. (A) Treatment of immortalized nasopharyngeal epithelial cells by TGFß. TGFß suppressed p63 expression in the early passage of immortalization (PD 76). (B) Nasopharyngeal carcinoma cells treated with TGFß. Treatment of nasopharyngeal carcinoma cells with TGFß could neither induce differentiation nor suppress p63 expression.

the cancer cell lines examined. As reviewed by p51B antibody, the TAp63 protein level did not show significant change after treatment with ATRA (Fig. 10B). ATRA is used in the treatment of head and neck cancer. Immortalized nasopharyngeal epithelial cells, NP460hTERT, were highly responsive to growth inhibitory action by ATRA as revealed in DNA synthesis study (Fig. 10D, open columns). Growth of cancer cells could also be inhibited by ATRA treatment, but the inhibition was much less compared to that in the immortalized nasopharyngeal epithelial cells (Fig. 10D, filled columns). The higher response of NP460hTERT cells to growth inhibition by ATRA may be related to more efficient downregulation of p63 expression. Inhibition of p63 expression may affect the survival and growth of squamous epithelial cells.

Suppression of p63 was associated with apoptosis in immortalized nasopharyngeal epithelial cells. We then examined the expression p63 in immortalized nasopharyngeal epithelial cells undergoing apoptosis using staurosporin. Staurosporin causes apoptosis in many types of cells, including epithelial cells by inhibition of protein kinase C (23). Treatment with $1 \mu \mathrm{M}$ of staurosporin for $6 \mathrm{~h}$ resulted in upregulation of cleaved caspase- 3 and cleaved PARP in the immortalized nasopharyngeal epithelial cells suggestive of onset of apoptosis (Fig. 11). Expression of p63 was suppressed 
A
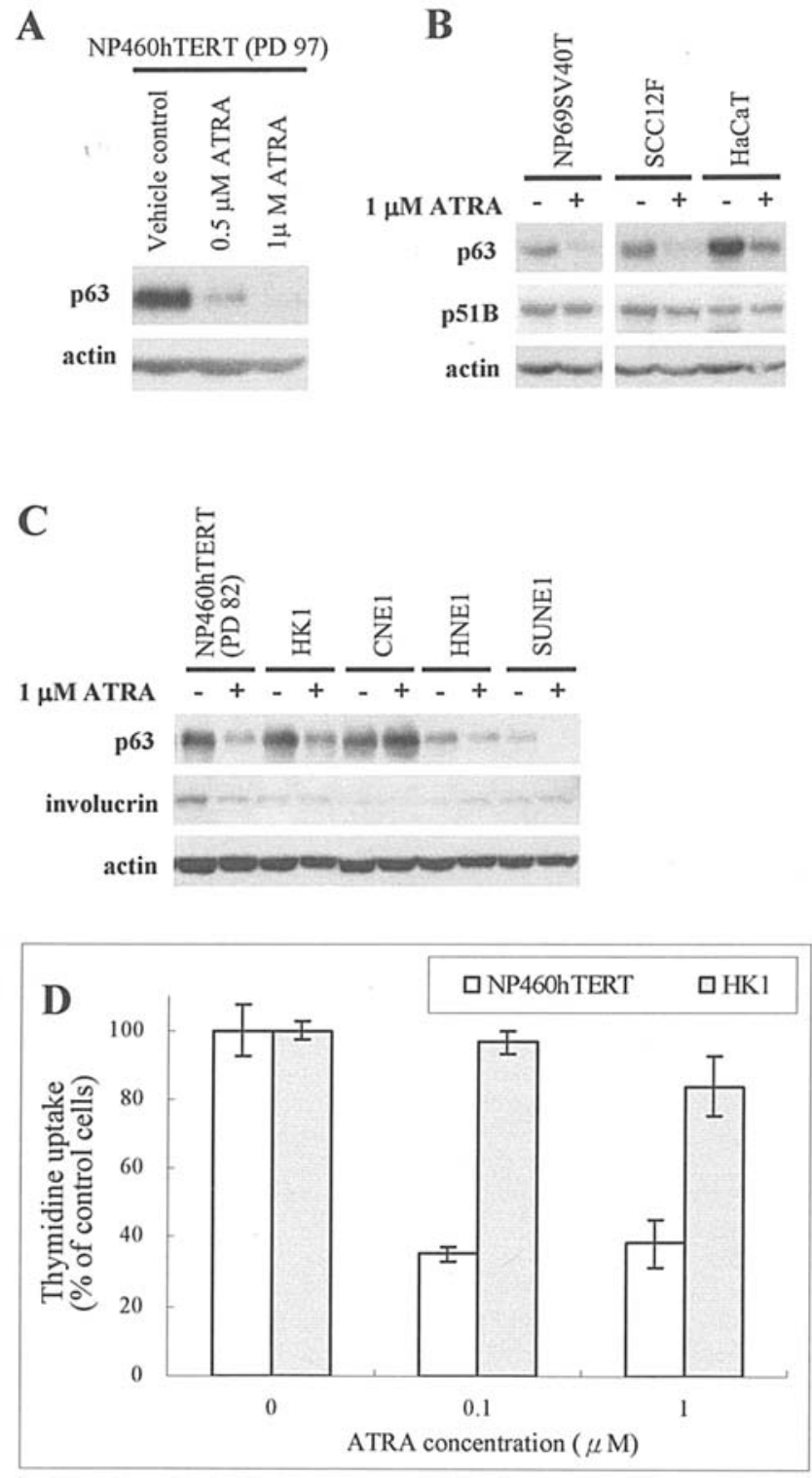

Figure 10. Expression of p63 in nasopharyngeal cells treated with all-trans retinoic acid (ATRA). (A) Treatment of immortalized nasopharyngeal epithelial cells with ATRA could lead to dose dependent suppression of p63. (B) Cells treated with ATRA had a reduction in p63 expression, but expression of TAp63, as indicated by the expression of p51B antibody, was not altered. (C) Nasopharyngeal epithelial cells and nasopharyngeal carcinoma cells treated with ATRA. The expression of p63 was suppressed after the ATRA treatment. (D) Relative DNA synthesis of ATRA treated immortalized epi-thelial cells (NP460hTERT) and cancer cells (HK1) measured by thymidine uptake. Thymidine uptake of cells without ATRA treatment was the control. NP460hTERT (open columns) was highly responsive to growth inhibition action of ATRA. Cancer cells were also inhibited by ATRA treatment in thymidine uptake experiment (filled columns). However, the sensitivity was lower than in NP460hTERT cells.

in cells undergoing apoptosis. We further examined if p63 may play a role in supporting cell survival by knocking down $\triangle \mathrm{Np} 63$ isoform in a cancer cell line, CNE2, using siRNA (si-p63). The p63 expression was lowered upon transfection of si-p63 into CNE2 cells. Interestingly, apoptosis was observed in si-p63 transfected CNE2 cells but not in control cells transfected with siRNA against GFP (si-GFP). Knocking

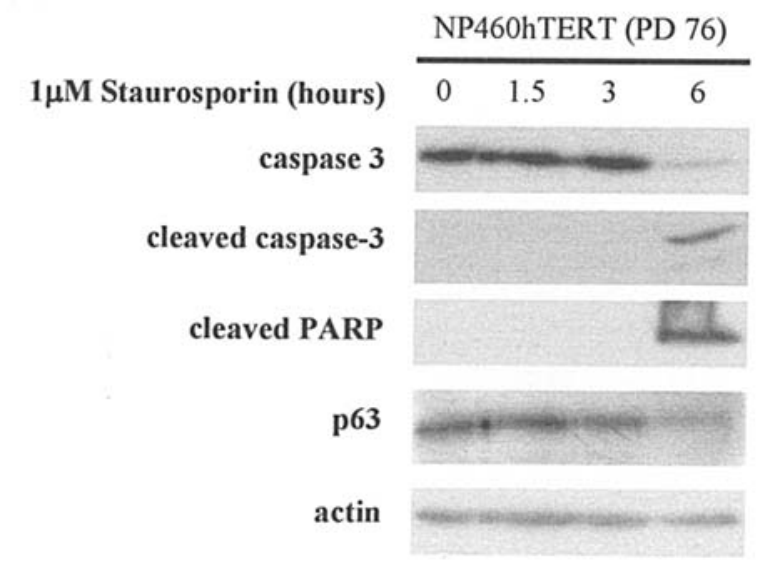

Figure 11. Expression of p63 in immortalized nasopharyngeal epithelial cells during apoptosis. NP460hTERT was subjected to staurosporin-induced apoptosis. The p63 expression was downregulated after induction of apoptosis indicated by the expression of cleaved caspase- 3 and cleaved PARP.

down $\triangle \mathrm{Np} 63$ in CNE2 cells resulted in an increase in p53, cleaved caspase-3 and cleaved PARP in apoptotic pathway (Fig. 12A). These results supported a role of $\Delta \mathrm{Np} 63$ in cell survival. In addition, knocking down of $\Delta \mathrm{Np} 63$ also resulted in downregulation of several adhesion molecules, integrin B4, laminin $\gamma-2$ and EGFR in CNE2 cells (Fig. 12B). The levels of Bcl-2 family proteins (Bcl-2, Bcl-xL and Bax), however, were not affected after $\Delta \mathrm{Np} 63$ knock-down (Fig. 12C).

Expression of 4 Np63 could drive clonal proliferation of nasopharyngeal epithelial cells. In order to study if $\Delta \mathrm{Np} 63$ could support proliferation of primary nasopharyngeal epithelial cells, we cloned the $\Delta \mathrm{Np} 63 \alpha$ from HK1 (a NPC cell line) by PCR amplification and expressed it via retroviral vector into primary cultures of non-malignant nasopharyngeal epithelial cells (Fig. 13). The $\Delta$ Np63a gene from nasopharyngeal carcinoma was cloned into the pLpLNCX retroviral vector. Successful cloning and expression of $\Delta \mathrm{Np} 63 \alpha$ in cells was confirmed by expression in HEK293 cells (Fig. 13A). Expression of $\Delta \mathrm{Np} 63 \alpha$ was able to drive clonal expansion of primary NP460 cells (Fig. 13B). However, expression of $\triangle \mathrm{Np} 63 \alpha$ alone was insufficient to immortalize the NP460 cells. Additional events notably inactivation of the $\mathrm{p} 16 / \mathrm{Rb}$ may be required to achieve immortalization of primary nasopharyngeal epithelial cells (11).

\section{Discussion}

It is intriguing that mutation of p53, which is common in human cancer, is rarely observed in nasopharyngeal carcinoma (9). It has long been postulated that alternative ways of inactivation of p53 may exist in nasopharyngeal carcinoma cells. The p63 may be a candidate gene expressed in nasopharyngeal carcinoma to inactivate the function of $\mathrm{p} 53$ gene. p63 is restrictedly expressed in epithelial cells of stratified epithelia, including bladder, esophagus, kidney, prostate, skin, thymus, tonsil, trachea and uterus $(17,24)$. It is expressed 
$\mathbf{A}$

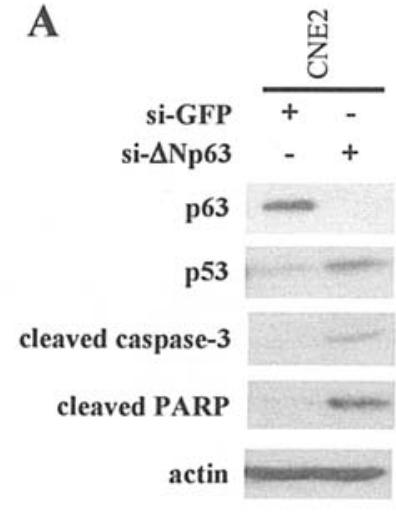

C

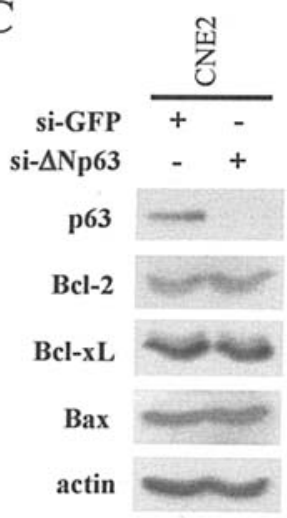

only in basal cell and squamous cell carcinomas, but not in adenocarcinomas (17). The p63 isoforms can be divided into two classes, a full-length transactivating class (TAp63) and a $\mathrm{NH}_{2}$-terminal transactivation domain truncated class $(\Delta \mathrm{Np} 63)$. The TAp63 isoform induces apoptosis and may have similar function to the wild-type p53. On the contrary, the $\Delta \mathrm{Np} 63$ cannot transactivate the $\mathrm{p} 53$ responsive genes and may function as a dominant negative gene to modulate p53 function in cells (3). Overexpression of $\Delta \mathrm{Np} 63$ may inactivate the p53 function and predispose the cells to cancer development. Possibly, the $\Delta \mathrm{Np} 63$ is a potential candidate to neutralize the action of p53 in nasopharyngeal carcinoma cells. There are many reports showing that the $\Delta \mathrm{Np} 63$ isoform exists at a higher level in cancer cells than normal cells $(8,16)$. Overexpression of $\Delta \mathrm{Np} 63$ is common in squamous cell carcinoma (25-28). In an earlier study, the expression of $\Delta \mathrm{Np} 63$ was observed to be located at the basal and suprabasal layers of the nasopharyngeal epithelium, which are proliferative and contain stem cells for regeneration of the nasopharyngeal epithelium (8). The expression of $\Delta \mathrm{Np} 63$ may interfere with the growth inhibitory action of the p53 protein to allow cell proliferation to take place.

Immortalization is an early step involved in carcinogenesis. The involvement of p63 in cell immortalization has not yet been explored. Similar to the findings in other head and neck squamous cell carcinomas (6) as well as squamous carcinoma of lung (5), $\Delta \mathrm{Np} 63 \alpha$ isoform is the predominat p63 transcript expressed in nasopharyngeal epithelial cells. Our results showed that $\Delta \mathrm{Np} 63 \alpha$ is also the predominant transcript expressed in primary and immortalized nasopha-

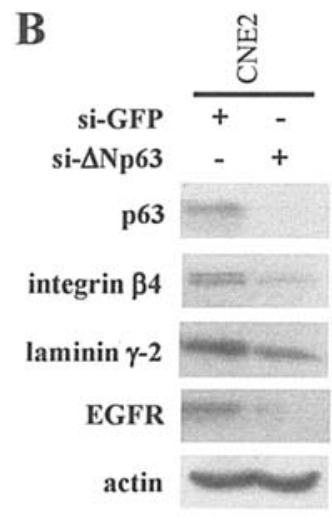

Figure 12. Effect of knocking down $\Delta \mathrm{Np} 63$ in cell proliferation and cell adhesion. (A) Decrease in $\Delta \mathrm{Np} 63$ could lead to upregulation of $\mathrm{p} 53$, cleaved caspase- 3 and cleaved PARP, which are implicated in cell death pathway. (B) Lower p63 expression resulted in reduction of cell adhesion and decreased EGFR expression. (C) Downregulation of p63 could not affect the expression levels of Bcl-2 family proteins.

ryngeal epithelial cells in active proliferation stage. This agrees with the role of $\mathrm{p} 63$ in the development and maintenance of epithelial structures. The p63 knock-out mice were born without limbs and skin $(3,27-29)$. The p63 was shown to upregulate Jagged 1 and activate the Notch signaling pathway in normal development (30). Nevertheless, the regulation of p63 expression in nasopharyngeal epithelial cells has not been examined. Immortalized nasopharyngeal epithelial cells may represent a premalignant stage and examination of regulation of p63 will have implication on the pathogenesis of nasopharyngeal carcinoma. In this study, expression of p63 was observed to be suppressed when the nasopharyngeal epithelial cells entered senescence and stopped proliferation. It was reactivated again when the cells escape from senescence and achieve immortalization status. The expression of p63 appears to have selective growth advantage and is selected in stably-immortalized nasopharyngeal epithelial cells and most nasopharyngeal carcinoma cells. A previous study showed that inhibition of p63 expression in $\Delta \mathrm{Np} 63 \alpha$ overexpressing cells led to apoptosis (31). Consistent with this study, we also observed that knocking down of $\Delta \mathrm{Np} 63$ suppressed cell growth together with upregulation of p53 and increase apoptotic events in CNE2 cells, thus supporting a role of $\Delta \mathrm{Np} 63$ in cell survival. The expression of $\Delta \mathrm{Np} 63$ is a general property of nasopharyngeal epithelial cells immortalized by different agents, including telomerase (hTERT) and viral oncogenes (SV40T and HPV16E6E7). The expression of $\Delta \mathrm{Np} 63$ closely reflects the proliferative status of the immortalized cells and increases at later stage of immortalization when cells have attained a stable proliferation stage. We have also observed that re-expression of $\Delta \mathrm{Np} 63$ resulted in clonal proliferation of nasopharyngeal epithelial cells, which is in line with a role of $\Delta \mathrm{Np} 63$ in proliferation. The role of $\Delta \mathrm{Np} 63$ in proliferation is supported by its roles as a positive regulator of the $\beta$-catenin signaling pathway (28)

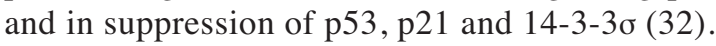

On the other hand, proliferation per se is not sufficient for the expression of $\Delta \mathrm{Np} 63$. Expression of $\Delta \mathrm{Np} 63$ was 
A
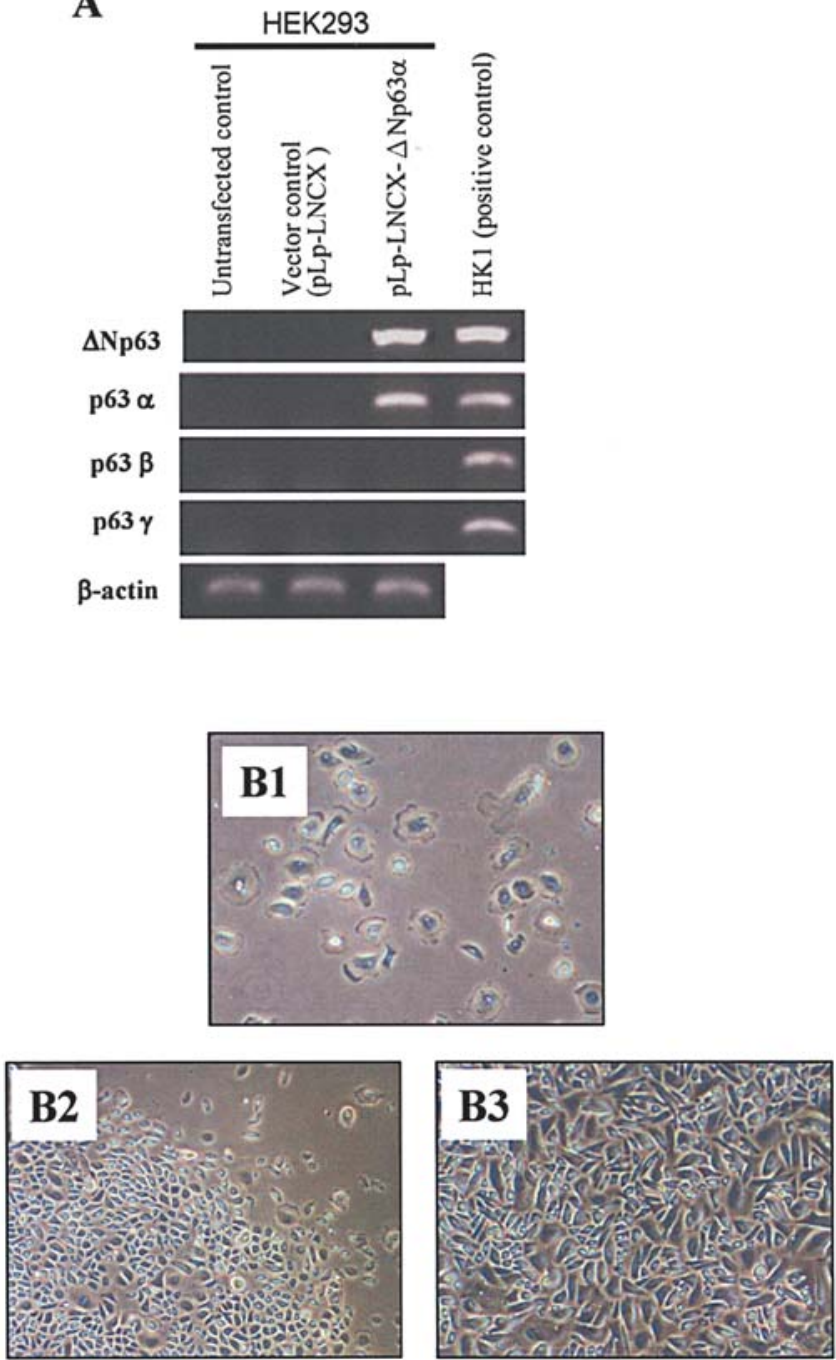

Figure 13. Cloning and expression of $\Delta \mathrm{Np} 63$ in nasopharyngeal epithelial cells. $\triangle \mathrm{Np} 63 \alpha$ amplified from the HK1 cDNA was cloned into pLp-LNCX retroviral vector. (A) HEK293 cells after transfection with pLp-LNCX$\Delta \mathrm{Np} 63 \alpha$ were subjected to RT-PCR. The pLp-LNCX- $\Delta$ Np63 $\alpha$ increased the level of $\Delta \mathrm{Np} 63 \alpha$ specifically. (B) Transfection of pLp-LNCX empty vector or pLp-LNCX- $\triangle \mathrm{Np} 63 \alpha$ into nasopharyngeal epithelial cells derived from primary culture (NP460). NP460 transfected with pLp-LNCX empty vector (B1) (x240) or with pLp-LNCX- $\triangle \mathrm{Np} 63 \alpha$ [B2 (x160) and B3 (x240)]. The expression of $\Delta \mathrm{Np} 63 \alpha$ led to clonal proliferation of the primary cultures (B2).

restricted to our immortalized cell system of nasopharyngeal and esophageal epithelial cells, and not observed in immortalized human ovarian surface epithelial (HOSE) and ovarian carcinoma cells. This showed that expression of $\Delta \mathrm{Np} 63$ is restricted to proliferative epidermal cells derived from ectodermal and endodermal origins and not in cells derived from mesodermal origin. However, induction of terminal differentiation of epidermal cells effectively suppresses the expression of $\Delta \mathrm{Np} 63$. The suppression of p63 expression is also observed in the terminally differentiated cells in the stratified squamous nasopharyngeal epithelium (8).

At present, the upstream events regulating expression of p63 is poorly understood. In this study, induction of terminal differentiation in culturing nasopharyngeal epithelial cells by serum or TGFß was shown to be the most effective in turning off expression of p63, indicating its close association with the differentiation status of nasopharyngeal epithelial cells.

In contrast, the cancer cells are commonly defective in response to the induction of differentiation by serum and TGFß and continue to express high level of p63 in the presence of serum in culture environment. In addition, immortalized cells at later passage, which has lost the sensitivity to TGFß, also have higher expression of p63. Expression of p63, in particular the $\Delta \mathrm{Np} 63$ isoform, may facilitate immortalization of nasopharyngeal epithelial cells and may be an early event in pathogenesis of nasopharyngeal carcinoma.

Another effective agent to suppress p63 expression identified in this study is retinoic acid. Retinoic acid can inhibit p63 in immortalized nasopharyngeal epithelial cells and cancer cell lines. Retinoic acid can regulate squamous differentiation and play an important function in maintaining the balance between proliferation and differentiation (33). The significance of retinoic acid in the regulation of p63 is unclear at this stage. Retinoic acid can inhibit in vitro growth of thyroid cancer cell lines (34). In addition, retinoic acid has been used in the treatment of cancer, such as oral leukoplakia (35). In this study, we showed that p63 could be downregulated by retinoic acid treatment and the degree of downregulation was correlated to the degree of growth inhibition. This agreed with the finding that knocking down p63 expression could induce apoptotic response. However, some cancer cells could acquire resistance to inhibitory effect of retinoic acid on p63 expression and growth. It would be interesting to determine if high expression level of p63 may confer resistance to growth inhibition by retinoic acid treatment in squamous carcinoma.

In conclusion, the observation of higher level of p63 expression, notably the $\Delta \mathrm{Np} 63$ isoform in immortalized nasopharyngeal epithelial cells indicated that it may be an early event selected for immortalization. Expression of $\Delta \mathrm{Np} 63$ is closely associated with cell proliferation. It could be effectively inhibited upon terminal differentiation of nasopharyngeal epithelial cells. Serum, TGFß and retinoic acid effectively inhibited its expression. Downregulation of $\Delta$ Np63 inhibits survival and induces apoptosis in cancer cells. Our study supports a role of expression of $\Delta \mathrm{Np} 63$ in pathogenesis of nasopharyngeal carcinoma.

\section{Acknowledgements}

This study was sponsored by the funding support received from the Research Grants Council, Hong Kong (HKU7457/ 04M), and a CRCG grant received from the University of Hong Kong.

\section{References}

1. Arrowsmith $\mathrm{CH}$ : Structure and function in the p53 family. Cell Death Differ 6: 1169-1173, 1999.

2. Waltermann A, Kartasheva NN and Dobbelstein M: Differential regulation of p63 and p73 expression. Oncogene 22: 5686-5693, 2003.

3. Yang A, Kaghad M, Caput D and McKeon F: On the shoulders of giants: p63, p73 and the rise of p53. Trends Genet 18: 90-95, 2002 . 
4. Okuyama R, Ogawa E, Nagoshi H, et al: p53 homologue, p51/p63, maintains the immaturity of keratinocyte stem cells by inhibiting Notch1 activity. Oncogene 26: 4478-4488, 2007.

5. Massion PP, Taflan PM, Jamshedur Rahman SM, et al: Significance of p63 amplification and overexpression in lung cancer development and prognosis. Cancer Res 63: 7113-7121, 2003.

6. Thurfjell N, Coates PJ, Uusitalo T, et al: Complex p63 mRNA isoform expression patterns in squamous cell carcinoma of the head and neck. Int J Oncol 25: 27-35, 2004.

7. Lo KW, To KF and Huang DP: Focus on nasopharyngeal carcinoma. Cancer Cell 5: 423-428, 2004.

8. Crook T, Nicholls JM, Brooks L, O'Nions J and Allday MJ: High level expression of deltaN-p63: a mechanism for the inactivation of p53 in undifferentiated nasopharyngeal carcinoma (NPC)? Oncogene 19: 3439-3444, 2000.

9. Lo KW, Mok CH, Huang DP, et al: p53 mutation in human nasopharyngeal carcinomas. Anticancer Res 12: 1957-1963, 1992.

10. Tsao SW, Wang X, Liu Y, et al: Establishment of two immortalized nasopharyngeal epithelial cell lines using SV40 large T and HPV16E6/E7 viral oncogenes. Biochim Biophys Acta 1590: 150-158, 2002.

11. Li HM, Man C, Jin Y, et al: Molecular and cytogenetic changes involved in the immortalization of nasopharyngeal epithelial cells by telomerase. Int J Cancer 119: 1567-1576, 2006.

12. Lo AK, Huang DP, Lo KW, et al: Phenotypic alterations induced by the Hong Kong-prevalent Epstein-Barr virus-encoded LMP1 variant (2117-LMP1) in nasopharyngeal epithelial cells. Int J Cancer 109: 919-925, 2004.

13. Lo AK, Liu Y, Wang XH, et al: Alterations of biologic properties and gene expression in nasopharyngeal epithelial cells by the Epstein-Barr virus-encoded latent membrane protein 1. Lab Invest 83: 697-709, 2003.

14. Deng W, Tsao SW, Guan XY, et al: Distinct profiles of critically short telomeres are a key determinant of different chromosome aberrations in immortalized human cells: whole-genome evidence from multiple cell lines. Oncogene 23: 9090-9101, 2004.

15. Tsao SW, Mok SC, Fey EG, et al: Characterization of human ovarian surface epithelial cells immortalized by human papilloma viral oncogenes (HPV-E6E7 ORFs). Exp Cell Res 218: 499-507, 1995.

16. Yang A, Kaghad M, Wang Y, et al: p63, a p53 homolog at 3q27-29, encodes multiple products with transactivating, deathinducing, and dominant-negative activities. Mol Cell 2: 305-316, 1998.

17. Di Como CJ, Urist MJ, Babayan I, et al: p63 expression profiles in human normal and tumor tissues. Clin Cancer Res 8: 494-501, 2002.

18. Green H, Easley K and Iuchi S: Marker succession during the development of keratinocytes from cultured human embryonic stem cells. Proc Natl Acad Sci USA 100: 15625-15630, 2003

19. Huang DP, Ho JH, Poon YF, et al: Establishment of a cell line (NPC/HK1) from a differentiated squamous carcinoma of the nasopharynx. Int J Cancer 26: 127-132, 1980.
20. Koster MI and Roop DR: The role of p63 in development and differentiation of the epidermis. J Dermatol Sci 34: 3-9, 2004.

21. De Laurenzi V, Rossi A, Terrinoni A, et al: p63 and p73 transactivate differentiation gene promoters in human keratinocytes. Biochem Biophys Res Commun 273: 342-346, 2000.

22. Tu CL, Chang W, Xie Z and Bikle DD: Inactivation of the calcium sensing receptor inhibits E-cadherin-mediated cell-cell adhesion and calcium-induced differentiation in human epidermal keratinocytes. J Biol Chem 283: 3519-3528, 2008.

23. Andersson M, Sjostrand J, Petersen A, Honarvar AK and Karlsson JO: Caspase and proteasome activity during staurosporin-induced apoptosis in lens epithelial cells. Invest Ophthalmol Vis Sci 41: 2623-2632, 2000

24. Vincek V, Knowles J, Li J and Nassiri M: Expression of p63 mRNA isoforms in normal human tissue. Anticancer Res 23: 3945-3948, 2003.

25. $\mathrm{Hu} \mathrm{H}, \mathrm{Xia} \mathrm{SH}, \mathrm{Li} \mathrm{AD}$, et al: Elevated expression of p63 protein in human esophageal squamous cell carcinomas. Int J Cancer 102: 580-583, 2002.

26. King KE, Ponnamperuma RM, Yamashita T, et al: DeltaNp63 alpha functions as both a positive and a negative transcriptional regulator and blocks in vitro differentiation of murine keratinocytes. Oncogene 22: 3635-3644, 2003.

27. Moll UM and Slade N: p63 and p73: roles in development and tumor formation. Mol Cancer Res 2: 371-386, 2004.

28. Patturajan M, Nomoto S, Sommer M, et al: DeltaNp63 induces beta-catenin nuclear accumulation and signaling. Cancer Cell 1: 369-379, 2002

29. Melino G, Lu X, Gasco M, Crook T and Knight RA: Functional regulation of p73 and p63: development and cancer. Trends Biochem Sci 28: 663-670, 2003.

30. Sasaki Y, Ishida S, Morimoto I, et al: The p53 family member genes are involved in the Notch signal pathway. J Biol Chem 277: 719-724, 2002.

31. Rocco JW, Leong CO, Kuperwasser N, DeYoung MP and Ellisen LW: p63 mediates survival in squamous cell carcinoma by suppression of p73-dependent apoptosis. Cancer Cell 9: 45-56, 2006

32. Westfall MD, Mays DJ, Sniezek JC and Pietenpol JA: The Delta Np63 alpha phosphoprotein binds the p21 and 14-3-3 sigma promoters in vivo and has transcriptional repressor activity that is reduced by Hay-Wells syndrome-derived mutations. Mol Cell Biol 23: 2264-2276, 2003.

33. Morgunkova AA: The p53 gene family: control of cell proliferation and developmental programs. Biochemistry (Mosc) 70: 955-971, 2005.

34. Elisei R, Vivaldi A, Agate L, et al: All-trans-retinoic acid treatment inhibits the growth of retinoic acid receptor beta messenger ribonucleic acid expressing thyroid cancer cell lines but does not reinduce the expression of thyroid-specific genes. J Clin Endocrinol Metab 90: 2403-2411, 2005.

35. Hong WK, Endicott J, Itri LM, et al: 13-cis-retinoic acid in the treatment of oral leukoplakia. N Engl J Med 315: 1501-1505, 1986. 